\begin{tabular}{|c|c|}
\hline Title & A study on benefit estimation that considers the values of travel time and travel time reliability in road networks \\
\hline Author(s) & Kato, T.; Uchida, K. \\
\hline Citation & $\begin{array}{l}\text { Transportmetrica. A, Transport science, 14(1-2), 89-109 } \\
\text { https://doi.org/10.1080/23249935.2017.1321695 }\end{array}$ \\
\hline Issue Date & 2018-01 \\
\hline Doc URL & http:/hdl.handle.net/2115/72255 \\
\hline Rights & $\begin{array}{l}\text { This is an A ccepted Manuscript of an article published by Taylor \& Francis in Transportmetrica A : Transport Science } \\
\text { on Jan Feb 2018, avail lable online: http:/wwww.tandfonline.com/10.1080/23249935.2017.1321695. }\end{array}$ \\
\hline Type & article (author version) \\
\hline File Information & manuscript1.pdf \\
\hline
\end{tabular}

Instructions for use 


\title{
A STUDY ON BENEFIT ESTIMATION THAT CONSIDERS THE VALUES OF TRAVEL TIME AND TRAVEL TIME RELIABILITY IN ROAD NETWORKS
}

\author{
T. KATO $^{\mathrm{a}}$ and K. UCHIDA ${ }^{\mathrm{b}}$ \\ ${ }^{a}$ Graduate School of Engineering, Hokkaido University, Japan \\ Email: ieppet@eng.hokudai.ac.jp \\ ${ }^{b}$ Graduate School of Engineering, Hokkaido University, Japan \\ Email: uchida@eng.hokudai.ac.jp
}

\begin{abstract}
This study proposes a benefit estimation method that considers travel time reliability. The proposed method is based on a network model that is formulated as a utility maximization problem with constraints. Since this utility maximization problem has the same equilibrium conditions as a multiclass user equilibrium traffic assignment problem with elastic demand, both transport demand forecasting and benefit estimation can be carried out in the same framework. By assuming a certain form for the utility function, the road network model can estimate the prohibitive price, so the proposed method is convenient for estimating opportunity loss due to disruption of origin-destination connection in the event of a natural disaster. Furthermore, the values of travel time and travel time reliability are estimated endogenously in the proposed method; thus, changes in these values can be reflected in the benefit estimation. A numerical experiment demonstrates the method presented in this study.
\end{abstract}

Key words: benefit estimation, value of travel time, value of travel time reliability, road network

\section{INTRODUCTION}

Cost-benefit analysis is widely used for determining an option that maximizes a benefit while reducing a cost. Benefit estimation is a main part of cost-benefit analysis. The benefit of a transport project is defined as the willingness to pay compensation for the welfare change generated by the project. Accurate benefit estimation is required for valid decision-making. Recently, travel time reliability is increasingly recognized as an important factor in benefit estimation of transport projects. Under travel time variability, travellers do not take only mean travel time into account; they also take travel time reliability into account. They allocate more time for their trip in order to reduce the possibility of late arrival. Therefore, reducing travel time variability generates a user benefit. Two tasks are involved in measuring the benefit from transport projects in road networks in terms of travel time reliability: evaluation of travel time reliability in the network and establishing the monetary value of travel time reliability. A benefit estimation method that is consistent with the evaluation of travel time reliability is also required.

For evaluating travel time reliability in a network, many studies have proposed equilibrium models that address uncertainties in the network. These uncertainties can be categorized into main two groups: those of demand, and those of supply. Lo \& Tung (2003) and Lo et al. (2006) proposed user equilibrium (UE) traffic assignment models that regard link capacity as a uniform distributed random variable. In contrast, some UE models based on random variations in demand have been proposed. Watling (2002) considered the demand variability generated from the route choice proportion. Clark \& Watling (2005) extended this model to consider stochastic origin-destination (O-D) demand flow when that flow follows a Poisson distribution. Furthermore, some authors considered both stochastic demand and supply in calculating stochastic travel time. Siu \& Lo (2008) proposed an equilibrium model that considered both stochastic O-D demand and link capacity. Lam et al. (2008) proposed an 
equilibrium model considering both stochastic O-D demand and the influence of adverse weather on link capacity.

Some studies have proposed equilibrium models with exogenously specified travel time distribution. These studies focused on the driver's risk-taking behavior. Based on a scheduling delay concept, Watling (2006) proposed a UE traffic assignment model that penalised late arrival. This concept is employed in the scheduling model. Chen \& Zhou (2010) proposed mean-excess traffic equilibrium models to capture aspects of reliability (on-time arrival) and unreliability (late arrival) of travel time variability. Ng et al. (2011) presented a method for assessing travel time reliability without specification of probability distributions of the sources of uncertainty.

Studies which address the value of travel time based on a utility maximization problem began with Becker (1965) and Deserpa (1973). Some analytical models which address the value of travel time reliability based on the utility maximization principle have been proposed. As shown in Fosgerau \& Karlström (2010), there are basically two mainstream approaches: the mean-variance approach, and the scheduling approach. Because of the simple structure of the mean-variance approach, many empirical studies have used it to estimate the value of travel time reliability (e.g., Lam \& Small, 2001; Brownstone \& Small, 2005). A scheduling approach was proposed by Small (1982) in which the traveller's cost function is assumed to depend on travel time and on the difference between the arrival time and the preferred arrival time.

Fosgerau \& Karlström (2010) developed a model that estimates the value of travel time variability based on scheduling preferences. By using a simple formulation of scheduling utility, they showed that the maximal expected utility is linear in the mean and standard deviation of trip duration. Fosgerau \& Engelson (2011) considered the value of travel time variability under scheduling preferences that were defined in terms of linearly time-varying utility rates associated with being at the origin and at the destination. They showed that a related measure of travel time variability is variance of travel time. Hjorth et al. (2015) empirically estimated scheduling functions and compared them with a more general form and a conventional form with regard to model fit by using SP data.

The studies that address travel time reliability based on equilibrium network models have required exogenously given values (preferences) of travel time and travel time reliability. In contrast, studies that addressed the value of travel time and of travel time reliability based on utility maximization have not addressed traveller route choice behavior in the network, even though it is an essential factor in estimating these two values. Although these two types of studies are closely related to each other, they have not been considered in the same framework. To address this issue, Uchida $(2012,2014)$ proposed two network models that estimate the values of travel time and travel time reliability. The two models were derived from a utility maximization problem under budget constraints. The models were finally formulated as UE traffic assignment problems with elastic demand in which the risk-averse driver route choice behavior was expressed. Kato et al. (2013) extended these network models to address heterogeneous drivers. The drivers in their model are distinguished by the value they place on travel time and on time reliability. This model was formulated as a multi-class UE traffic assignment problem with elastic demand.

For evaluating the benefit of transport projects, traffic demand forecasting and benefit estimation consistent with microeconomics are required. To forecast traffic demand, a UE model or a stochastic user equilibrium (SUE) model is adopted. These models consider equilibrium conditions in which the driver minimizes the disutility of a trip. That disutility is expressed as a function of the traffic demand that expresses congestion in a road network. Although disutility minimization behavior is expressed in terms of UE or SUE, no traffic demand forecasting model has been formulated as a utility maximization problem that uses traffic demand as the decision variable while expressing flowdependent disutility. Therefore, existing benefit estimation methods for transport projects have two steps: transport demand forecasting, and benefit estimation. For example, Kidokoro (2004) estimated the benefit by expressing route choice behavior as a utility maximization problem for a representative consumer. However, the equilibrium user disutility of a trip was given exogenously by using the 
results of a demand forecasting model. Since these two steps are carried out separately in existing benefit estimation methods, the lack of consistency between them has been discussed in the literature. Kidokoro (2006) clarified the relationship between assumptions made in transport-demand modeling and benefit estimation methods by focusing on the forms of the utility function of a representative consumer. However, a benefit estimation method that carries out both steps in the same framework has not been proposed. In the literature on transport demand forecasting methods that include travel time reliability, many measures of travel time variability have been used, such as standard deviation, variance and percentile. The estimated benefit is biased when there is mismatch between the travel time variability measure in transport demand forecasting and that in benefit estimation. A benefit estimation method that can carry out both steps in the same framework is useful for correcting this bias.

Ueda et al. (2002) indicated that the prohibitive price of transport at which a traveler in a transport network abandons his/her trip plays an important role in both estimating the benefit of newly opened transport service and developing a new pricing scheme. In the literature, there are no studies that address a finite value of prohibitive price within a utility maximization framework. To evaluate an opportunity loss that results from the disruption of an O-D connection in the event of a natural disaster, it is necessary to estimate the finite value of the prohibitive price.

Kono \& Morisugi (2000) and Jiang \& Morikawa (2004) examined the changes in value of travel time by static analysis on travel and individual socio-economic environments. In the logit model, the form of the traffic demand function is significantly affected by the values of travel time and travel time reliability. Therefore, these values also affect the benefit estimated by using the logit model. However, the logit model cannot address the change in value of travel time and travel time reliability. Existing studies have not addressed how these changes could be reflected in benefit analysis.

In the present study, we propose a benefit estimation method that is based on the network model proposed by Kato et al. (2013), which can carry out the two steps in the same framework. In this model, the inverse demand function, which describes the relationship between the generalized travel cost of an origin-destination (O-D) pair and the corresponding O-D traffic demand, i.e., the relationship between the price of the O-D pair trip and the corresponding O-D traffic demand, is derived based on the Stone-Geary utility function. Therefore, the prohibitive price of each O-D pair is estimated as a positive finite value. In addition, the proposed method does not require predefined values (preferences) of travel time and travel time reliability. Therefore, by using the proposed method, we can estimate the benefits considering the changes in the values of travel time and travel time reliability.

To summarize, in this paper we propose a method of benefit estimation for transport projects that considers travel time reliability. Since the proposed method is based on the network model proposed by Kato et al. (2013), it has the following convenient properties: (i) both transport demand forecasting and benefit estimation can be carried out in the same framework, (ii) the prohibitive price can be estimated as a positive finite value, and (iii) the benefits considering the changes in the values of travel time and travel time reliability can be estimated.

The paper is structured as follows. In Section 2, we review the network model proposed by Kato et al. (2013). Section 3 presents a benefit estimation method based on the network model described in Section 2. In Section 4, a numerical experiment is carried out on a test network. Finally, the conclusion and directions for future study are given in Section 5.

\section{THE MODEL}

In this section, we review the network model proposed by Kato et al. (2013) as preliminary to explaining the method of benefit estimation. For a detailed discussion, please refer to Kato et al. (2013). 


\subsection{Notations}

In this study, we consider the same random variables as employed by Kato et al. (2013): stochastic O$\mathrm{D}$ demand flow, and link capacity. The following notations are used for representing network flows in this paper.

A

Set of links in the network

$\mathrm{I}(|\mathrm{I}|)$

Set of O-D pairs in the network

$\mathrm{J}_{i}\left(\left|\mathrm{~J}_{i}\right|\right)$

Set of routes between O-D pair $i$

$\delta_{a j}$

Variable that equals 1 if link $a$ is part of route $j$, and 0 otherwise

$Q_{i}$

Stochastic traffic demand for O-D pair $i$

$q_{i} \quad$ Mean traffic demand for O-D pair $i$

$F_{i j} \quad$ Stochastic flow on route $j$ for O-D pair $i$

$f_{i j}$

Mean flow on route $j$ for O-D pair $i\left(q_{i}=\sum_{j \in J_{i}} f_{i j} \forall i \in \mathrm{I}\right)$

$V_{a} \quad$ Stochastic flow on link $a$

$v_{a} \quad$ Mean flow on link $a\left(v_{a}=\sum_{i \in \mathrm{I}} \sum_{j \in J_{i}} \delta_{a j} \cdot f_{i j} \forall a \in \mathrm{A}\right)$

Mean flow that passes through both links $a$ and $b\left(v_{a b}=\sum_{i \in \mathrm{I}} \sum_{j \in J_{i}} \delta_{a j} \cdot \delta_{b j} \cdot f_{i j} \cdot \forall a, b \in \mathrm{A}\right.$; if $a=b, v_{a b}=v_{a}$ )

\subsection{Model Structure}

In the model, the following three assumptions for the estimation were employed.

A1. Route flows in the network can be observed or estimated. Note that, link flows and O-D demands can be calculated by route flows. Considering recent advances in ITS technology, it may be possible to obtain large amounts of link flow data in a road network. By applying an OD flow estimation technique that uses a partial set of observed link flows (e.g., Sherali et al., 2003), O-D flows and route flows in the network can be estimated, as can link flows. The observed or estimated flow can be regarded as the mean flow.

A2. Observed or estimated traffic flows are generated from the optimal behavior of each driver in the network such that the utility level of each driver is maximized subject to the three budget constraints regarding mean travel time, mean travel cost and travel time reliability. In general econometrics, it is also assumed that the observed behavior is generated by the optimal behavior, i.e., utility maximization behavior.

A3. The upper bounds for the three budget constraints in A2 can be calculated by using observed or estimated traffic flows. As shown in Kato et al. (2013), the left-hand side of the three budget constraints can be expressed by the link performance function in which the explanatory variable is link flow. Following A2, the observed flows maximize utility and satisfy the constraints. Therefore, if we know the link performance functions, then the right-hand side of (2)-(4) can be calculated by substituting the observed flows into the left-hand side of (2)-(4). The link performance function can be estimated by calibrating parameters in the function from link performance data.

Note that, by applying the model proposed by Kato et al. (2013) and by employing A1-A3, the values of (mean) travel time and of travel time reliability can be estimated from observed traffic flows. That is, we regard the observed traffic flows to contain drivers' revealed preferences of travel time, travel cost and travel time reliability in a road network. In addition, the price and the demand of goods other than transportation demand are assumed to be fixed.

The network model was formulated as the following primary problem [PP]: 


$$
v=\max u\left(q_{1}, \ldots, q_{|I|}\right)=\sum_{i \in \mathrm{I}} \int_{0}^{q_{i}} \frac{\alpha_{i}}{w+1} \cdot d w
$$

s.t.

$$
\begin{gathered}
t_{i} \cdot q_{i}=\phi_{i} \forall i \in \mathrm{I} \\
\rho_{i} \cdot q_{i}=\pi_{i} \forall i \in \mathrm{I} \\
\sigma_{i} \cdot q_{i}=\theta_{i} \forall i \in \mathrm{I} \\
q_{i}+e_{i}=q_{i} \forall i \in \mathrm{I}
\end{gathered}
$$

It should be noted that the decision variables in PP are $q_{i}(\forall i \in \mathrm{I})$, although $q_{i}(\forall i \in \mathrm{I})$ are assumed to be observed (A1). By solving PP with parameters estimated from observed flows, we can obtain not only the observable flows but also the values of travel time and travel time reliability in the traffic situation observed. The objective function $u$ in (1) can be regarded as a direct utility function (Varian ,1992) in the network, since the objective function becomes

$$
u\left(q_{1}, \ldots, q_{|I|}\right)=\sum_{i \in \mathrm{I}} \int_{0}^{q_{i}} \frac{\alpha_{i}}{w+1} \cdot d w=\sum_{i \in \mathrm{I}} \alpha_{i} \cdot \ln \left(q_{i}+1\right)
$$

(6) follows the style of a Stone-Geary utility function (Stone, 1954) by assuming that the mean O-D flow, $q_{i}$, is the number of substitute trips $i$, and that $\alpha_{i}(>0)$ is the marginal propensity to consume such that $\sum_{i} \alpha_{i}=1$. The inequality constraints shown by (2) to (4) can be regarded as budget constraints in which three different prices $\left(t_{i}, \rho_{i}\right.$ and $\sigma_{i}$ ) are offered to the O-D flow $q_{i}$. $e_{i}\left(0 \leq e_{i}<q_{i}\right)$ in (5) is excess demand (Gartner, 1980) that does not appear in the network. $q_{i}$ in (5) is a constant given to each O-D pair $i$ that is larger than the supposed maximal traffic demand. The utility function (6) means that the greater the O-D flow is, the higher the utility level is. However, these three budget constraints do not allow the O-D flows to increase independently of the observed link flows. A calibration method of the marginal propensity to consume $\alpha_{i}$ will be discussed later. $t_{i}$ is the price for mean travel time between O-D pair $i$. In a similar way, $\rho_{i}$ and $\sigma_{i}$ are prices for mean travel cost and travel time reliability between O-D pair $i$, respectively. Note that the values of $t_{i}, \rho_{i}$ and $\sigma_{i}$ are calculated by using the network flows. Following A3, the upper bounds for three budget constraints that are respectively denoted by $\phi_{i}, \pi_{i}$ and $\theta_{i}$ are calculated by using the observed route flows. $t_{i}, \rho_{i}$ and $\sigma_{i}$ are the functions of network flows.

If the marginal propensity to consume, $\alpha_{i}$, which is the proportion of supernumerary income spent on substitute trip $i$, is known, then we can obtain the relationship between the demand $q_{i}$ and three different prices $\left(t_{i}, \rho_{i}\right.$ and $\sigma_{i}$ ). However, we assume that $\alpha_{i}$ is constant in predicting the demand and price, since the change in $\alpha_{i}$ cannot be predicted in advance. Hence, $\alpha_{\mathrm{i}}$ can be considered as the expenditure share of substitute trip $i . \alpha_{\mathrm{i}}$ can be calculated as

$$
\alpha_{i}=\frac{\phi_{i}+\pi_{i}+\theta_{i}}{\sum_{k \in I}\left(\phi_{k}+\pi_{k}+\theta_{k}\right)} \quad \forall i \in I
$$

As shown by Kato et al. (2013), each of the three upper bounds $\phi_{i}, \pi_{i}$ and $\theta_{i}$ for the three constraints shown by (2) to (4) can be transformed to an integration of a corresponding function that is either mean travel time, travel cost, or travel time reliability of O-D pair $i$ with respect to network flows. By applying the partial Lagrangian with respect to the budget constrains denoted by (2) to (4), we can obtain the equilibrium conditions that are equivalent to multi-class UE traffic assignment problems with elastic demand. The equilibrium conditions for the route flows are expressed as (8).

$$
f_{i j} \cdot\left(c_{i j}(\mathbf{F})-d_{i}\left(e_{i}\right)\right)=0, f_{i j} \geq 0, c_{i j}(\mathbf{F})-d_{i}\left(e_{i}\right) \geq 0
$$

where 


$$
\begin{gathered}
c_{i j}(\mathbf{F})=\lambda_{i}^{*} \cdot E\left[\Xi_{i j}\right]+\omega_{i}^{*} \cdot E\left[\Gamma_{i j}\right]+\gamma_{i}^{*} \cdot \operatorname{var}\left[\Xi_{i j}\right] \\
d_{i}\left(e_{i}\right)=\frac{\alpha_{i}}{q_{i}-e_{i}+1}
\end{gathered}
$$

In (8), $c_{i j}(\mathbf{F})$ is the route performance value in which $\mathbf{F}$ is the vector of route flows, and $d_{i}\left(e_{i}\right)$ is the inverse demand function that provides the minimum route performance value for O-D pair $i$. In (9), $\Xi_{i j}$ and $\Gamma_{i j}$ are stochastic travel time and stochastic travel cost on route $j$ for O-D pair $i$, respectively. Variance of route travel time is employed as an index of travel time variability in (9). Note that other indexes, such as standard deviation, can be applied to the present formulation. (Uchida, 2012, 2014 and Kato et al., 2013 have adopted variance of route travel time as the index of travel time variability.) $\lambda_{i}^{*}, \omega_{i}^{*}$ and $\gamma_{i}^{*}$ are respectively the optimal Lagrangian multipliers for constraints (2) to (4). $\left(\lambda_{i}^{*}, \omega_{i}^{*}\right.$ and $\gamma_{i}^{*}$ are also the same as the shadow prices of the mean travel time, mean travel cost and travel time reliability of O-D pair $i$, respectively.) Hence, the value of travel time (VOT) and of travel time reliability $(V O R)$ for O-D pair $i$ can be obtained as $V O T_{i}=\lambda_{i}^{*} / \omega_{i}^{*}$ and $V O R_{i}=\gamma_{i}^{*} / \omega_{i}^{*}$, respectively. By using the values of travel time and travel time reliability, we can obtain the generalized cost, which is a monetary cost experienced by a traveler making a given trip in a network. When the flow on O-D pair $i$ is $0\left(q_{i}-e_{i}=0\right)$, the inverse demand function equals $\alpha_{i}$. Therefore, the prohibitive price for O-D pair $i$ is $\alpha_{i}$. By dividing the route performance value by the optimal Lagrangian multiplier of travel cost, $\omega_{i}^{*}$, the corresponding prohibitive generalized travel cost is calculated as $\alpha_{i} / \omega_{i}^{*}$.

How to check the accuracy of the estimated results should be mentioned. Since a set of observed flows are considered and are assumed to be correct in the models proposed by Uchida $(2012,2014)$ and by Kato et al. (2013), there is no way to evaluate the accuracy of the estimated values other than calculating the corresponding error, e.g., root-mean-square error between the observed flows and the estimated flows. In the case where several sets of observed flows are available, a maximum likelihood estimation method can be applied. The accuracy of the estimated O-D demands and the values of travel time and travel time reliability can be examined based on an inverse hessian matrix of the loglikelihood function. Accordingly, the accuracy of the benefit estimation proposed in this paper can be discussed in this case.

In the maximum likelihood estimation framework, the upper bounds $\phi_{i}, \pi_{i}$, and $\theta_{i}(\forall i \in \mathrm{I})$ for the constraints shown by (2) to (4), which are inputs to the network model proposed by Kato et al. (2013), are also decision variables. Since the network model has the same structure as the multi-user class UE, network flows are estimated by solving the network model with the given inputs. Therefore, the likelihood function that corresponds to the model inputs can be calculated by using the several sets of observed flows. By applying a perturbed Fischer-Burmeister function (Fukushima, 1998), the optimal conditions of the PP, which was transformed as a nonlinear complementary problem in Kato et al. (2013), can be expressed as equality constraints. Therefore, we can formulate the maximum likelihood estimation problem with equilibrium constraints in which the decision variables are the three upper bounds, O-D demands, and the values of travel time and travel time reliability.

\section{THE METHOD OF BENEFIT ESTIMATION}

The benefit of a transport project is defined as the willingness to pay compensation for the welfare change generated by the project. Therefore, the benefit is measured as a monetary value. By calculating the changes of the generalized cost and the traffic demand generated by the transport project, we can estimate the benefit. As an intuitive example, we consider the simple case in which the O-D demand and the values of travel time and travel time reliability are fixed. When the mean and variance of travel time decrease by one unit as a result of a transport project, the benefit of the project is given as the sum of one unit value of travel time and travel time reliability. It should be emphasized 
that we take the change of O-D demand and the value of travel time reliability into account in the benefit estimation method proposed in this study.

In this section, we estimate the expenditure function from PP for the purpose of estimating compensating variation (CV), which is an index of benefits. By solving PP without and with the update of the network parameters generated by transport projects, the three different corresponding prices $\left(t_{i}, \rho_{i}\right.$ and $\left.\sigma_{i}\right)$ at the equilibrium can be obtained. We denote these three prices at the equilibrium as $t_{i}^{*}, \rho_{i}^{*}$ and $\sigma_{i}^{*}$, respectively. We employ the same convention for all the variables in the rest of paper. It is reasonable to assume that these three prices $\left(t_{i}^{*}, \rho_{i}^{*}\right.$ and $\left.\sigma_{i}^{*}\right)$ are constants by focusing on the equilibrium condition for benefit estimation. By using these three equilibrium prices, the expenditure function can be obtained as shown below.

It was assumed that all the equality constraints denoted by (2) to (4) are satisfied under the equilibrium condition. This assumption shows that the consumption of goods other than the amount of substitute trips does not change after a transportation project and that the drivers in the network generate as many trips as possible within the budget constraints. The former assumption can be relaxed by introducing goods other than traffic demand. For example, it can be relaxed by applying a nested constant elasticity substitution utility function in which the consumption of goods other than the amount of substitute trips is decided in an upper-level problem, whereas the amount of substitute trips is decided in a lower-level problem. The latter assumption is the same one as employed in typical time allocation models (Becker, 1965, Deserpa, 1973). Since the right-hand sides of (2) to (4), $\phi_{i}, \pi_{i}$ and $\theta_{i}$, are calculated by using observed flows, this assumption holds when the link flows are the same as the observed ones. For benefit estimation, we have to consider the changes in link flows generated by transport projects. Following again this assumption, new Lagrangian multipliers corresponding to the constraints will be obtained after the transport projects. Therefore, the values of travel time and travel time reliability will change as a result of the transport projects. We obtain the following relationship at the equilibrium by applying this assumption.

$$
\begin{aligned}
\frac{\left(t_{i}^{*}+\rho_{i}^{*}+\sigma_{i}^{*}\right) \cdot q_{i}^{*}}{\sum_{k \in I}\left(t_{k}^{*}+\rho_{k}^{*}+\sigma_{k}^{*}\right) \cdot q_{k}^{*}} & =\frac{\phi_{i}+\pi_{i}+\theta_{i}}{\sum_{k \in I}\left(\phi_{k}+\pi_{k}+\theta_{k}\right)} \\
& =\alpha_{i} \quad \forall i \in I
\end{aligned}
$$

Note that the limit of (11) still holds as $q_{i}^{*}$ approaches zero from the right.

Based on the assumption mentioned above and (11), the inequality constraints denoted (2) to (4) can be unified as the following equality constraint.

$$
\sum_{i \in \mathrm{I}} p_{i} \cdot q_{i}=\sum_{i \in \mathrm{I}} \psi_{i}
$$

where

$$
\begin{array}{ll}
p_{i}=t_{i}^{*}+\rho_{i}^{*}+\sigma_{i}^{*} & \forall i \in \mathrm{I} \\
\psi_{i}=\phi_{i}+\pi_{i}+\theta_{i} & \forall i \in \mathrm{I}
\end{array}
$$

We consider then the optimization problem in which the utility function denoted by (1) is maximized subject to the constraints denoted by (12) and (5). In the following paper, we call this optimization problem "PP-2". Note that, PP-2 is equivalent to PP, as shown in Appendix A. Assuming that the O-D flow, $q_{i}$, is the number of substitute trips $i$, it is reasonable to regard $p_{i}$ as the price and $\psi_{i}$ as the budget of substitute trip $i$.

By solving PP-2, the demand function can be obtained as shown by (15). This demand function is the same as that obtained by employing a Cobb-Douglas style utility function (Cobb \& Douglas, 1928).

$$
q_{i}=\frac{\alpha_{i} \cdot \sum_{k \in I} \psi_{k}}{p_{i}} \forall i \in I
$$


By substituting the demand function into the utility function denoted by (1), the indirect utility function, $v$, is obtained, as shown in (16).

$$
v=\sum_{i \in \mathrm{I}} \alpha_{i} \cdot \ln \left(\frac{\alpha_{i} \cdot \sum_{k \in I} \psi_{k}}{p_{i}}+1\right)
$$

According to duality theory, the expenditure function is obtained as

$$
e(\mathbf{p}, v)=-\sum_{i \in I} p_{i}+\exp (v) \cdot \prod_{i \in I}\left(\frac{p_{i}}{\alpha_{i}}\right)^{\alpha_{i}}
$$

$\mathbf{p}$ in (17) is the vector of the prices of O-D flows $E\left[Q_{i}\right]$. Therefore, the CV is obtained as follows.

$$
C V=e\left(\mathbf{p}^{w}, v^{w}\right)-e\left(\mathbf{p}^{w}, v^{w o}\right)=\left\{\exp \left(v^{w}\right)-\exp \left(v^{w o}\right)\right\} \cdot \prod_{i \in I}\left(\frac{p_{i}^{w}}{\alpha_{i}}\right)^{\alpha_{i}}
$$

The superscripts $w$ and wo indicate the condition with the transport projects and without the transport projects, respectively. We employ these conventions for all kinds of variables in the rest of paper. Note that, $p_{i}$ is different from the generalized travel cost of O-D pair $i$. However, by applying the following one-to-one mapping from the price, $p_{i}$, to the corresponding generalized travel cost, $g c_{i}$, which is derived from (8) to (11), $p_{i}$ can be transformed to $g c_{i}$. (The correspondence to the case of $q_{i}^{*}=0$ was discussed in Section 2.)

$$
g c_{i}=\frac{1}{\omega_{i}^{*}} \cdot\left(d\left(e_{i}\right)\right)=\left\{\begin{array}{ccc}
\frac{1}{\omega_{i}^{*}} \cdot\left(\frac{p_{i}}{\sum_{k \in I} \psi_{k}} \cdot \frac{q_{i}^{*}}{q_{i}^{*}+1}\right) & \text { if } & q_{i}^{*}>0 \\
\alpha_{i} / \omega_{i}^{*} & \text { if } & q_{i}^{*}=0
\end{array}\right.
$$

\section{NUMERICAL EXPERIMENTS}

\subsection{Benefit estimation by using the proposed method}

\subsubsection{Settings}

In this section, the observed route flows are assumed to hold for the UE conditions under the predefined values of travel time and travel time reliability. The route performance function obtained based on the BPR function is employed. Figure-1 shows a test network with two O-D pairs. For each O-D pair, the number of equilibrated routes is four, as shown by the sequence of link numbers $1 \rightarrow 4 \rightarrow 5 \rightarrow 8, \quad 1 \rightarrow 4 \rightarrow 6 \rightarrow 7,2 \rightarrow 3 \rightarrow 5 \rightarrow 8$ and $2 \rightarrow 3 \rightarrow 6 \rightarrow 7$ for O-D pair 1 , and $9 \rightarrow 11 \rightarrow 5 \rightarrow 14$, $9 \rightarrow 11 \rightarrow 12 \rightarrow 13,10 \rightarrow 3 \rightarrow 5 \rightarrow 14$, and $10 \rightarrow 3 \rightarrow 12 \rightarrow 13$ for O-D pair 2. Only two links, 3 and 5, are shared by the two classes of drivers, i.e., the driver traveling O-D pair 1 and the driver traveling O-D pair 2. Note that drivers traveling different O-D pairs are assumed to have different preferences on route choice, and thus multi-class users are expressed in this manner.

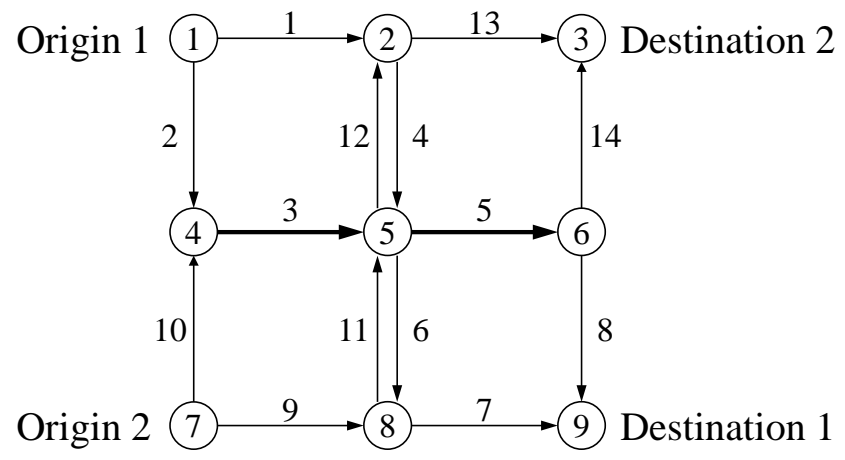


Figure-1. Test Network

We assume the following mean travel time function, mean travel cost function, and function of covariance of two link travel times, which are respectively given by

$$
\begin{aligned}
& t_{a}^{\mathrm{t}}\left(v_{a}\right)=\frac{l_{a}}{s_{0}} \cdot\left(1+m \cdot\left(\frac{v_{a}}{c_{a}}\right)^{n}\right) \forall a \in \mathrm{A} \\
& \stackrel{\mathrm{t}}{\rho_{a}}\left(v_{a}\right)=p_{g} \cdot\left(r_{1} \cdot l_{a} \cdot\left(1+m \cdot\left(\frac{v_{a}}{c_{a}}\right)^{n}\right)+r_{2} \cdot l_{a}\right) \quad \forall a \in \mathrm{A} \\
& \stackrel{\mathrm{t}}{\sigma}_{a b}\left(v_{a b}\right)=\frac{g_{a}}{c_{a}} \cdot \frac{g_{b}}{c_{b}} \cdot\left(v_{a b}\right)^{h} \quad \forall a, b \in \mathrm{A}
\end{aligned}
$$

where

$$
\begin{gathered}
v_{a}=\sum_{i \in \mathrm{I}} \sum_{j \in J_{i}} \delta_{a j} \cdot f_{i j} \quad \forall a \in \mathrm{A} \\
v_{a b}=\sum_{i \in \mathrm{I}} \sum_{j \in \mathrm{J}_{i}} \delta_{a j} \cdot \delta_{b j} \cdot f_{i j} \cdot \forall a, b \in \mathrm{A}
\end{gathered}
$$

In (20), $c_{a} \forall a \in \mathrm{A}$ is the mean capacity of link $a$ that we set at $1.67[\mathrm{pcu} / \mathrm{min}$ ] for all links in the network, where pcu represents the passenger car unit; $l_{a} \forall a \in \mathrm{A}$ is the length of link $a$ that we set at $10[\mathrm{~km}] \forall a \in \mathrm{A} ; s_{0}$ is the free flow speed (1 [km/min]); and $m$ and $n$ are parameters of the BPR function ( $m=2.62, n=5$ ). In (21), $p_{g}$ is gas price (140 [JPY/l]); $r_{1}=0.009$ and $r_{2}=0.059$ are the parameters for travel cost function estimated in Uchida (2010). In (22), $g_{a} \forall a \in A$ and $h$ are the parameters for variance and covariance of travel time ( $\left.g_{a}=3 \forall a \in A, h=2\right)$. Note that we assume that the covariance of the two stochastic link travel times is expressed as a function of $v_{a b}$, which is the mean flow passing both links $a$ and $b$. The validity of this assumption is discussed in Appendix B. Mean route travel time and mean route travel cost are given by the sum of those of the links that compose the route, as shown in (25) to (26).

$$
\begin{gathered}
E\left[\Xi_{i j}\right]=\sum_{a \in \mathrm{A}} \mathrm{t}_{a}\left(v_{a}\right) \cdot \delta_{a j} \forall i \in \mathrm{I}, \forall j \in \mathrm{J}_{i} \\
E\left[\Gamma_{i j}\right]=\sum_{a \in \mathrm{A}} \stackrel{\mathrm{L}}{\rho}_{a}\left(v_{a}\right) \cdot \delta_{a j} \forall i \in \mathrm{I}, \forall j \in \mathrm{J}_{i}
\end{gathered}
$$

The variance of route travel time is given as follows. (See Appendix B for the derivation.)

$$
\operatorname{var}\left[\Xi_{i j}\right]=\sum_{a \in \mathrm{A} b \in \mathrm{A}} \hat{\sigma}_{a b}\left(v_{a b}\right) \cdot \delta_{a j} \cdot \delta_{b j} \forall i \in \mathrm{I}, \forall j \in \mathrm{J}_{i}
$$

The utility function of this problem is given by

$$
u\left(q_{1}, q_{2}\right)=\alpha_{1} \cdot \ln \left(q_{1}+1\right)+\alpha_{2} \cdot \ln \left(q_{2}+1\right)
$$

where $\left(\alpha_{1}, \alpha_{2}\right)$ are calculated as $(0.5,0.5)$. The equilibrated link flows that we regard as the observed link flows are calculated based on the predefined value of travel time, 40.00 [JPY/min]; and the value of travel time reliability, 10.00 [JPY/min ${ }^{2}$ ], as shown in Table- 1 . We set the same values of travel time and travel time reliability for each O-D pair for the purpose of seeing how these two values change according to the transport project.

Table- 1 . The observed link flows

\begin{tabular}{lcccccccccccccc}
\hline Link & 1 & 2 & 3 & 4 & 5 & 6 & 7 & 8 & 9 & 10 & 11 & 12 & 13 & 14 \\
\hline$E\left[V_{a}\right]$ & 2.10 & 1.23 & 2.48 & 2.10 & 2.48 & 2.10 & 2.10 & 1.23 & 2.10 & 1.23 & 2.10 & 2.10 & 2.10 & 1.23 \\
\hline
\end{tabular}

\subsubsection{Results}

As the first step, by solving PP with the observed flows, we estimate the values of travel time and travel time reliability for each O-D pair. Here, the values of travel time and travel time reliability for 
two classes users are respectively estimated as 40.00 [JPY/min] and 10.00 [JPY $/ \mathrm{min}^{2}$ ] for each O-D pair. Those values are same as those used for generating the observed flows. Table- 2 shows the O-D flows, the route flows and route performance values without the transport project. The corresponding link flows are shown in Table-3. The diagonal elements of Table-3 represent $v_{a}$, and the off-diagonal elements represent $v_{a b}$. It is shown that the flows, the value of travel time and the value of travel time reliability are all estimated consistently with the observed flows. Mean link travel time, mean link travel cost and covariance of two link travel times without the transport project are calculated by substituting the flows shown in Table-3 into (20) to (22), respectively.

Table-2. O-D flows, route flows and route performance values without the transport project

\begin{tabular}{|c|c|c|c|c|c|c|c|c|}
\hline O-D & \multicolumn{4}{|c|}{1} & \multicolumn{4}{|c|}{2} \\
\hline$E\left[Q_{i}\right][\mathrm{pcu} / \mathrm{min}]$ & & & & & & & & \\
\hline Route & 1 & 2 & 3 & 4 & 5 & 6 & 7 & 8 \\
\hline Link seq. & $1-4-5-8$ & $1-4-6-7$ & $2-3-5-8$ & $2-3-6-7$ & $\begin{array}{r}9-11- \\
5-14\end{array}$ & $\begin{array}{c}9-11- \\
12-13\end{array}$ & $\begin{array}{l}\text { 10-3- } \\
5-14\end{array}$ & $\begin{array}{c}10-3- \\
12-13\end{array}$ \\
\hline$E\left[F_{i j}\right][\mathrm{pcu} / \mathrm{min}]$ & 0.90 & 1.20 & 0.34 & 0.90 & 0.90 & 1.20 & 0.34 & 0.90 \\
\hline$E\left[\Xi_{i j}\right][\min ]$ & 87.4 & 83.4 & 91.5 & 87.4 & 87.4 & 83.4 & 91.5 & 87.4 \\
\hline \begin{tabular}{l|l|l}
$E$ & $\Gamma_{i j}$ & {$[\mathrm{JPY}]$}
\end{tabular} & 477.6 & 470.9 & 484.2 & 477.6 & 477.6 & 470.9 & 484.2 & 477.6 \\
\hline $\operatorname{var}\left[\Xi_{i j}\right]\left[\min ^{2}\right]$ & 50.1 & 67.0 & 33.2 & 50.1 & 50.1 & 67.0 & 33.2 & 50.1 \\
\hline$c_{i j}(\mathbf{F})$ [no unit] & 4,475 & 4,475 & 4,475 & 4,475 & 4,475 & 4,475 & 4,475 & 4,475 \\
\hline
\end{tabular}

Table-3. Mean link flows $v_{a}$ and $v_{a b}$ without the transport project

\begin{tabular}{c||c|c|c|c|c|c|c|c|c|c|c|c|c|c}
\hline Link & 1 & 2 & 3 & 4 & 5 & 6 & 7 & 8 & 9 & 10 & 11 & 12 & 13 & 14 \\
\hline \hline 1 & 2.10 & 0 & 0 & 2.10 & 0.90 & 1.20 & 1.20 & 0.90 & 0 & 0 & 0 & 0 & 0 & 0 \\
\hline 2 & $:$ & 1.23 & 1.23 & 0 & 0.33 & 0.90 & 0.90 & 0.33 & 0 & 0 & 0 & 0 & 0 & 0 \\
\hline 3 & & $:$ & 2.48 & 0 & 0.67 & 0.90 & 0.90 & 0.33 & 0 & 1.23 & 0 & 0.90 & 0.90 & 0.33 \\
\hline 4 & & & $:$ & 2.10 & 0.90 & 1.20 & 1.20 & 0.90 & 0 & 0 & 0 & 0 & 0 & 0 \\
\hline 5 & & & & $:$ & 2.48 & 0 & 0 & 1.23 & 0.90 & 0.33 & 0.90 & 0 & 0 & 1.23 \\
\hline 6 & & & & & $:$ & 2.10 & 2.10 & 0 & 0 & 0 & 0 & 0 & 0 & 0 \\
\hline 7 & & & & & & $:$ & 2.10 & 0 & 0 & 0 & 0 & 0 & 0 & 0 \\
\hline 8 & & & & & & & $:$ & 1.23 & 0 & 0 & 0 & 0 & 0 & 0 \\
\hline 9 & & & & & & & & $:$ & 2.10 & 0 & 2.10 & 1.20 & 1.20 & 0.90 \\
\hline 10 & & & & & & & & & $:$ & 1.23 & 0 & 0.90 & 0.90 & 0.33 \\
\hline 11 & & & & & & & & & & $:$ & 2.10 & 1.20 & 1.20 & 0.90 \\
\hline 12 & & & & & & & & & & & $:$ & 2.10 & 2.10 & 0 \\
\hline 13 & & & & & & & & & & & & $:$ & 2.10 & 0 \\
\hline 14 & & & & & & & & & & & & & $:$ & 1.23 \\
\hline
\end{tabular}

(All units are $[\mathrm{pcu} / \mathrm{min}]$ )

Next, we assume a transport project that increases the mean capacity of link 1 by 0.83 [pcu/min] (i.e., a change from 1.67 [pcu/min] to $2.5[\mathrm{pcu} / \mathrm{min}])$. By solving PP with the transport project, we estimate the equilibrium flows and the route performance values as shown in Table-4. Table-5 shows the corresponding link flows. As mentioned in Section 3, the values of travel time and travel time reliability can change as a result of the transport project. In this numerical experiment, we assumed that only the Lagrangian multipliers of travel time reliability change as a result of the transport project whereas those of mean travel time and mean travel cost remained unchanged. Therefore, only the value of travel time reliability is assumed to change as a result of the transport project. As a result, the 
values of travel time reliability are estimated as $9.00\left[\mathrm{JPY} / \mathrm{min}^{2}\right]$ for O-D pair 1 and as 10.13 $\left[\mathrm{JPY} / \mathrm{min}^{2}\right]$ for O-D pair 2 with the transport project.

Table-4. The O-D flows, the route flows and route performance values with the transport project

\begin{tabular}{|c|c|c|c|c|c|c|c|c|}
\hline \multirow{4}{*}{$\begin{array}{c}\text { O-D } \\
E\left[Q_{i}\right] \\
\text { [pcu/min }] \\
\text { Route }\end{array}$} & \multicolumn{4}{|c|}{1} & \multicolumn{4}{|c|}{2} \\
\hline & \multirow{2}{*}{\multicolumn{4}{|c|}{$\begin{array}{c}3.40 \\
(+0.068)\end{array}$}} & \multirow{2}{*}{\multicolumn{4}{|c|}{$\begin{array}{c}3.35 \\
(+0.017)\end{array}$}} \\
\hline & & & & & & & & \\
\hline & 1 & 2 & 3 & 4 & 5 & 6 & 7 & 8 \\
\hline Link seq. & 1-4-5-8 & $1-4-6-7$ & $2-3-5-8$ & 2-3-6-7 & $\begin{array}{r}9-11- \\
5-14\end{array}$ & $\begin{array}{r}9-11- \\
12-13\end{array}$ & $\begin{array}{r}10-3- \\
5-14\end{array}$ & $\begin{array}{r}10-3- \\
12-13\end{array}$ \\
\hline$\left.E \mid F_{i j}\right\rfloor$ & 0.99 & 1.28 & 0.28 & 0.86 & 0.87 & 1.19 & 0.36 & 0.93 \\
\hline [pcu/min] & $(+0.085)$ & $(+0.080)$ & $(-0.055)$ & $(-0.045)$ & $(-0.030)$ & $(-0.005)$ & $(+0.027)$ & $(+0.027)$ \\
\hline$E\left[\Xi_{i j}\right]$ & 86.9 & 83.3 & 90.4 & 86.8 & 87.0 & 82.7 & 90.7 & 86.4 \\
\hline [min] & $(-0.5)$ & $(-0.1)$ & $(-1.1)$ & $(-0.6)$ & $(-0.4)$ & $(-0.7)$ & $(-0.8)$ & $(-1.0)$ \\
\hline$\left.E \mid \Gamma_{i j}\right\rfloor$ & 476.7 & 470.8 & 482.5 & 476.6 & 477.0 & 469.9 & 483.0 & 476.0 \\
\hline [JPY] & $(-0.9)$ & $(-0.1)$ & $(-1.7)$ & $(-1.0)$ & $(-0.6)$ & $(-1.0)$ & $(-1.2)$ & $(-1.6)$ \\
\hline $\operatorname{var}\left\lfloor\Xi_{i j}\right\rfloor$ & 48.4 & 65.1 & 32.0 & 48.7 & 48.8 & 66.6 & 33.6 & 51.4 \\
\hline$\left[\mathrm{min}^{2}\right]$ & $(-1.7)$ & $(-1.9)$ & $(-1.2)$ & $(-1.4)$ & $(-1.3)$ & $(-0.4)$ & $(+0.4)$ & $(+1.3)$ \\
\hline$c_{i j}(\mathbf{F})$ & 4,387 & 4,387 & 4,387 & 4,387 & 4,453 & 4,453 & 4,453 & 4,453 \\
\hline [no unit] & $(-88)$ & $(-88)$ & $(-88)$ & $(-88)$ & $(-22)$ & $(-22)$ & $(-22)$ & $(-22)$ \\
\hline
\end{tabular}

$(\cdot)$ : The difference between without versus with the transport project

Table-5. Mean link flows $v_{a}$ and $v_{a b}$ with the transport project

\begin{tabular}{c||c|c|c|c|c|c|c|c|c|c|c|c|c|c}
\hline Link & 1 & 2 & 3 & 4 & 5 & 6 & 7 & 8 & 9 & 10 & 11 & 12 & 13 & 14 \\
\hline \hline 1 & 2.27 & 0 & 0 & 2.27 & 0.98 & 1.27 & 1.27 & 0.98 & 0 & 0 & 0 & 0 & 0 & 0 \\
\hline 2 & $:$ & 1.13 & 1.13 & 0 & 0.28 & 0.85 & 0.85 & 0.28 & 0 & 0 & 0 & 0 & 0 & 0 \\
\hline 3 & & $:$ & 2.43 & 0 & 0.65 & 0.85 & 0.85 & 0.28 & 0 & 1.28 & 0 & 0.93 & 0.93 & 0.37 \\
\hline 4 & & & $:$ & 2.27 & 0.98 & 1.27 & 1.27 & 0.98 & 0 & 0 & 0 & 0 & 0 & 0 \\
\hline 5 & & & & $:$ & 2.50 & 0 & 0 & 1.27 & 0.87 & 0.37 & 0.87 & 0 & 0 & 1.23 \\
\hline 6 & & & & & $:$ & 2.13 & 2.13 & 0 & 0 & 0 & 0 & 0 & 0 & 0 \\
\hline 7 & & & & & & $:$ & 2.13 & 0 & 0 & 0 & 0 & 0 & 0 & 0 \\
\hline 8 & & & & & & & $:$ & 1.27 & 0 & 0 & 0 & 0 & 0 & 0 \\
\hline 9 & & & & & & & & $:$ & 2.07 & 0 & 2.07 & 1.18 & 1.18 & 0.87 \\
\hline 10 & & & & & & & & & $:$ & 1.28 & 0 & 0.93 & 0.93 & 0.37 \\
\hline 11 & & & & & & & & & & $:$ & 2.07 & 1.18 & 1.18 & 0.87 \\
\hline 12 & & & & & & & & & & & $:$ & 2.12 & 2.12 & 0 \\
\hline 13 & & & & & & & & & & & & $:$ & 2.12 & 0 \\
\hline 14 & & & & & & & & & & & & & $:$ & 1.23 \\
\hline
\end{tabular}

(All units are [pcu/min])

As shown in Table-4, however, the route flows on routes 1 and 2, which include the link extended by the project, increase, and their route performance values decrease, as a result of the project. In contrast, both the route flows and the route performance values on routes 3 and 4 decrease. The reasons for these changes are that some drivers traveling on route 3 or 4 before the project change to route 1 or 2 after the project. Such driver route changes and the decrease in the value of travel time reliability for O-D pair 1 decrease the route performance values, thereby increasing the O-D demand. In total, the equilibrium route performance values for O-D pair 1 decrease. In O-D pair 2, the route flows on routes 7 and 8 increase, whereas those on routes 5 and 6 decrease. These changes are caused by the decrease 
in the flow on link 3, which contributes to traffic flows for both O-D pairs. The flow on link 3 of O-D pair 1 decreases by about 0.17 [pcu/min] (the sum of the decreases in flows on routes 1 and 2). Such route flow changes and the increase in the value of travel time reliability decrease the route performance values, thereby increasing the O-D demand. In total, the equilibrium route performance values for O-D pair 2 decrease.

Now, we have the equilibrium prices, $t_{i}^{*}, \rho_{i}^{*}$ and $\sigma_{i}^{*}$, without and with the transport project. Accordingly, we can reformulate PP as PP-2 as shown in Section 3. The prices of substitute trip $i, p_{i}$, with and without the transport project are obtained as shown in Table-6. By substituting them into the demand function shown by (15), the O-D flows are obtained as shown in Table-6. The number of substitute trips $i, q_{i}$, increase due to the decrease in the price of substitute trip, $p_{i}$, which is results from the transport project. Note that the O-D flows estimated by PP (shown in Table-4) and those estimated by PP-2 (shown in Table-6) are almost the same.

The benefits are generated from increase in the total utility of the network. Table- 6 shows CV estimated by (18) and Consumer Surplus (CS), which equals the user benefit in a special case (Willig, 1976). CS can be calculated by integrating the demand function with respect to $p_{i}$. Note that CV and CS shown in Table-6 are monetary values obtained by substituting the generalized travel cost, $g c_{i}$, shown by (19) into $p_{i}$ in (18).

Table-6. O-D demand, price of substitute trip, generalized cost, CV and CS

\begin{tabular}{|c|c|c|c|c|c|c|c|c|c|}
\hline O-D & $\begin{array}{c}E\left[Q_{i}\right]^{\text {wo }} \\
{[\mathrm{pcu} / \mathrm{min}]}\end{array}$ & $\begin{array}{c}E\left[Q_{i}\right]^{w} \\
{[\mathrm{pcu} / \mathrm{min}]}\end{array}$ & $p_{i}^{\text {wo }}$ & $p_{i}^{w}$ & $\begin{array}{c}g c_{i}^{\text {wo }} \\
\text { [JPY] }\end{array}$ & $\begin{array}{c}g c_{i}^{w} \\
\text { [JPY] }\end{array}$ & $\begin{array}{c}C V \\
{[\mathrm{JPY}]}\end{array}$ & $\begin{array}{c}C S_{i} \\
{[\mathrm{JPY}]}\end{array}$ & $\begin{array}{c}C S \\
{[\mathrm{JPY}]}\end{array}$ \\
\hline 1 & 3.33 & 3.40 & 18.20 & 17.80 & 74.58 & 73.12 & \multirow{2}{*}{370.37} & 298.05 & \multirow{2}{*}{372.65} \\
\hline 2 & 3.33 & 3.35 & 18.20 & 18.10 & 74.58 & 74.22 & & 74.62 & \\
\hline
\end{tabular}

\subsection{Comparison between the proposed method and existing methods}

\subsubsection{Settings}

As mentioned in the introduction, the proposed method can estimate the benefit while considering the changes in the values of travel time and travel time reliability. In this section, we compare the proposed model to existing methods. We adopt a Nguyen-Dupuis network (1984) with 4 O-D pairs, 25 routes and 19 directed links (Figure-2). The link sequences of the routes are shown in Table-7.

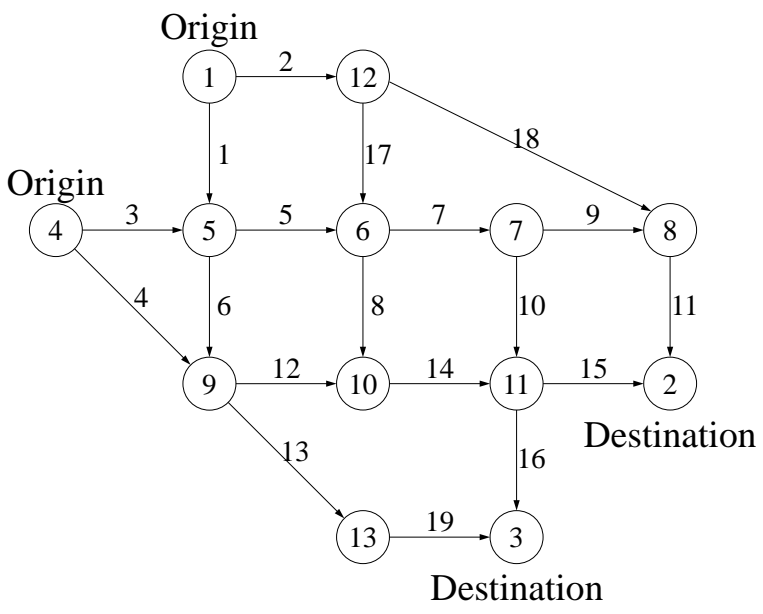

Figure-2. Nguyen-Dupuis Network

Table-7. Routes and link sequences

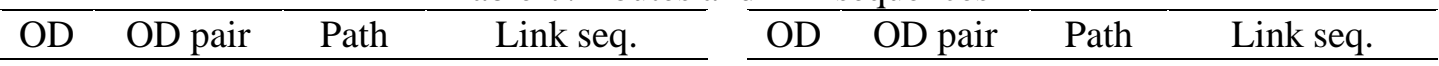




\begin{tabular}{|c|c|c|c|}
\hline \multirow{8}{*}{1} & \multirow{8}{*}{$1-2$} & 1 & $2-18-11$ \\
\hline & & 2 & $1-5-7-9-11$ \\
\hline & & 3 & 1-5-7-10-15 \\
\hline & & 4 & $1-5-8-14-15$ \\
\hline & & 5 & 1-6-12-14-15 \\
\hline & & 6 & 2-17-7-9-11 \\
\hline & & 7 & $2-17-7-10-15$ \\
\hline & & 8 & $2-17-8-14-15$ \\
\hline \multirow{5}{*}{2} & \multirow{5}{*}{$4-2$} & 9 & 4-12-14-15 \\
\hline & & 10 & $3-5-7-9-11$ \\
\hline & & 11 & $3-5-7-10-15$ \\
\hline & & 12 & $3-5-8-14-15$ \\
\hline & & 13 & $3-6-12-14-15$ \\
\hline
\end{tabular}

\begin{tabular}{llll}
\hline & & 14 & $1-6-13-19$ \\
& & 15 & $1-5-7-10-16$ \\
3 & 16 & $1-5-8-14-16$ \\
& $1-3$ & 17 & $1-6-12-14-16$ \\
& & 18 & $2-17-7-10-16$ \\
& 19 & $2-17-8-14-16$ \\
\hline \multirow{4}{*}{4} & 20 & $4-13-19$ \\
& & 21 & $4-12-14-16$ \\
& $4-3$ & 22 & $3-6-13-19$ \\
& & 23 & $3-5-7-10-16$ \\
& 24 & $3-5-8-14-16$ \\
& & 25 & $3-6-12-14-16$ \\
\hline
\end{tabular}

We assume the same mean travel time function, mean travel cost function and function of covariance of two link travel times as those of the first experiment. The parameters of these functions are also the same as those of the first experiment, excluding the link length, $l_{a} \forall a \in \mathrm{A}$, and the link capacities, $c_{a} \forall a \in \mathrm{A}$. Table-8 shows the link lengths and link capacities.

Table-8. Link length and capacities

\begin{tabular}{ccccccc}
\hline Link & $\begin{array}{c}\text { Link length } \\
l_{a}[\mathrm{~km}]\end{array}$ & $\begin{array}{c}\text { Link capacity } \\
C_{a}[\mathrm{pcu} / \mathrm{min}]\end{array}$ & & Link & $\begin{array}{c}\text { Link length } \\
l_{a}[\mathrm{~km}]\end{array}$ & $\begin{array}{c}\text { Link capacity } \\
C_{a}[\mathrm{pcu} / \mathrm{min}]\end{array}$ \\
\hline 1 & 10 & 25 & & 11 & 10 & 25 \\
2 & 10 & 25 & & 12 & 10 & 25 \\
3 & 10 & 25 & & 13 & 20 & 25 \\
4 & 20 & 25 & & 14 & 10 & 25 \\
5 & 10 & 25 & & 15 & 10 & 25 \\
6 & 10 & 25 & & 16 & 10 & 25 \\
7 & 10 & 25 & & 17 & 10 & 25 \\
8 & 10 & 25 & & 18 & 30 & 25 \\
9 & 10 & 25 & & 19 & 10 & 25 \\
\hline
\end{tabular}

The equilibrated link flows that can be regarded as observed link flows have been calculated based on the predefined value of travel time, 40.00 [JPY/min]; and the value of travel time reliability, 10.00 [JPY/min ${ }^{2}$ ]. The O-D flows without the transport project are assumed as 25 [pcu/min] for O-D pairs 1 and 4 and 16.17 [pcu/min] for O-D pairs 2 and 3.

\subsubsection{Results}

For the purpose of comparison, we consider three cases.

Case1. The change of traffic demand and the values of travel time and travel time reliability generated from a transport project are ignored. This case is one of the most simplified methods (the socalled "shortcut approach").

Case2. Only the changes of traffic demand generated from a transport project are taken into account; the values of travel time and travel time reliability do not change. This case corresponds to the combined model in which the parameter on the demand function is calculated from the utility maximization problem denoted by (1)-(5). However, this model does not consider the change of values of travel time and travel time reliability. It means that the constraints on travel time, travel cost and travel time reliability are ignored in estimating future travel demand. 
Case3. The change of traffic demand and the change of the value of travel time reliability generated from a transport project are both taken into account. This case corresponds to the method proposed in this study. Unlike Cases 1 and 2, this case takes the constraints about travel time, travel cost and travel time reliability into account in estimating the future travel demands.

Note that only the value of travel time reliability is assumed to change as a result of the transport project in this experiment, as mentioned in the first experiment. We evaluate the user benefit generated from a transport project in each case. We assume a transport project that gradually increases the mean capacity of link 8 from 25.00 to 33.33 [pcu/min] by 0.17 [pcu/min]. Note that CV cannot be evaluated in Cases 1 and 2, since the expenditure function cannot be obtained in both two cases. Then, we use CS to approximate the user benefit. Figure-3 shows the changes of CS in the three cases. As shown in Figure-3, the estimated benefit in Case 1 is the biggest, that in Case 2 is the middle and that in Case 3 is smallest. Since there is no induced traffic demand in Case 1, the generalized user costs decrease the most among the three cases. Thus the estimated benefit in Case 1 is the greatest among the three cases. On the other hand, induced traffic demand in Cases 2 Case 3 make the estimated benefit smaller than that in Case 1. In Case 3, the value of travel time reliability is estimated as 8.09 [JPY $/ \mathrm{min}^{2}$ ] for O-D pair 1, as $9.43\left[\mathrm{JPY} / \mathrm{min}^{2}\right]$ for O-D pair 2, as $7.95\left[\mathrm{JPY} / \mathrm{min}^{2}\right]$ for O-D pair 3, and as $9.25\left[\mathrm{JPY} / \mathrm{min}^{2}\right]$ for O-D pair 4, when the capacity of link 8 is 33.33 [pcu/min]. Decreases in the values of travel time reliability generate decreases in generalized user costs. Therefore, the increase in estimated benefit is diminished and the estimated benefit in Case 3 is the smallest among the three cases. When the mean capacity of link 8 is 33.33 [pcu/min], the O-D flows are estimated as 25.75, 17.12, 16.62, 24.91 [pcu/min] for each O-D pairs in Case2; 25.95, 17.12, 16.76, 24.92 [pcu/min] for each O-D pairs in Case3.

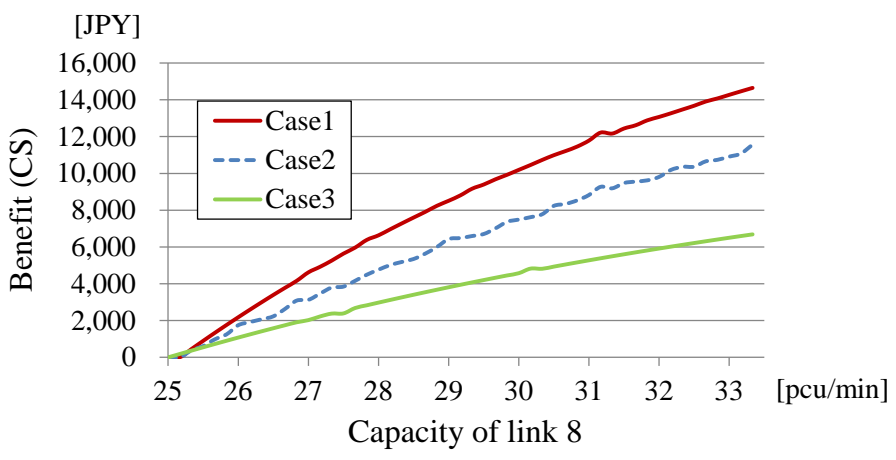

Figure-3. Estimated benefit in the three cases

\section{CONCLUDING REMARKS}

This study proposed a benefit estimation method that considers travel time reliability, based on a network model proposed by Kato et al. (2013). The network model was formulated as a utility maximization problem with constraints on (mean) travel time, travel cost, and travel time reliability. The proposed method assumes that the inequality constraints become equalities at the equilibrium, and that the marginal propensities to consume are constants. Applying these assumptions, we reformulated the network model as a standard utility maximization problem with a constraint, which allows the expenditure function to be obtained. Finally, CV can be estimated by the expenditure function. Since the network model has the same equilibrium conditions as a multi-class UE traffic assignment problem with elastic demand, the proposed method can achieve both transport demand forecasting and benefit estimation in the same framework. By using the proposed method, the values of travel time and travel time reliability are estimated endogenously. Furthermore, the method can evaluate benefits considering the changes in the values of travel time and travel time reliability. We provided a one-toone mapping from the price, $p_{i}$, to the corresponding generalized travel cost, $g c_{i}$. By applying this mapping, a benefit estimated based on the price, $p_{i}$ with no unit, can be easily transformed to a benefit estimated based on the generalized cost, $g c_{i}$ with a monetary unit. In addition, the prohibitive prices of each O-D pair can be transformed to monetary values. Therefore, the opportunity loss due to disruption of O-D connection in the event of a natural disaster is easily estimated. 
Two numerical experiments were carried out to demonstrate the proposed benefit estimation method. In the first experiment, we introduced a test network that has two O-D pairs and 4 routes in each O-D pair with symmetrical topology. We assumed a transport project that increased the road capacity of a link in the network. The positive benefits from the transport project were estimated as CV. We assumed that the value of travel time reliability could change as a result of the transport project, whereas the value of travel time remained unchanged. It was found that the value of travel time reliability of drivers on the O-D pair that includes the link extended by the project decreases whereas the value of travel time reliability of drivers on the other O-D pair increases. A detailed analysis of the changes in the value of travel time reliability, i.e., comparative statics, needs to be carried out. In the second experiment, we verified differences between the results of the proposed method and those of existing methods. The Nguyen-Dupuis network (1984) was adopted in this experiment. It can be considered that the differences in the results are great enough to affect decision making.

In the proposed benefit method, we made the relatively strong assumption that the budget constraints remain unchanged without or with the transport project. This assumption can be relaxed by introducing goods other than transport demand and substitution elasticities between transport demand and those other goods. These are our future tasks. We proposed the essential idea of a new benefit estimation method. As the next step, we have to consider how this method can be applied to real-life cases. The numerical experiments only applied the proposed methods for a small network. A more efficient algorithm is required for the proposed method to be applied to a real-size network. Furthermore, the current framework cannot fully evaluate the accuracy of the estimated results. As discussed in Section 2, it can be solved by applying the maximum likelihood estimation. We will present it in the near future.

\section{APPENDIX A}

As shown in Section 2, we can solve PP by applying the partial Lagrangian with respect to the constraints denoted by (2) to (4). The Lagrangian function for the utility maximization problem at the equilibrium is

$$
L^{*}=\sum_{i \in \mathrm{I}} \alpha_{i} \cdot \ln \left(q_{i}^{*}+1\right)+\sum_{i \in \mathrm{I}}\left\{\lambda_{i}^{*} \cdot\left(\phi_{i}-t_{i}^{*} \cdot q_{i}^{*}\right)+\omega_{i}^{*} \cdot\left(\pi_{i}-\rho_{i}^{*} \cdot q_{i}^{*}\right)+\gamma_{i}^{*} \cdot\left(\theta_{i}-\sigma_{i}^{*} \cdot q_{i}^{*}\right)\right\}
$$

The second term on the right-hand side of (29) can be expressed as

$$
\sum_{i \in \mathrm{I}}\left[\left\{\frac{\lambda_{i}^{*} \cdot \phi_{i}+\omega_{i}^{*} \cdot \pi_{i}+\gamma_{i}^{*} \cdot \theta_{i}}{\phi_{i}+\pi_{i}+\theta_{i}} \cdot\left(\phi_{i}+\pi_{i}+\theta_{i}\right)\right\}-\left\{\frac{\lambda_{i}^{*} \cdot t_{i}^{*}+\omega_{i}^{*} \cdot \rho_{i}^{*}+\gamma_{i}^{*} \cdot \sigma_{i}^{*}}{t_{i}^{*}+\rho_{i}^{*}+\sigma_{i}^{*}} \cdot\left(t_{i}^{*}+\rho_{i}^{*}+\sigma_{i}^{*}\right) \cdot q_{i}^{*}\right\}\right]
$$

Since the equality constraints are assumed at the equilibrium, we obtain

$$
\begin{aligned}
\frac{\lambda_{i}^{*} \cdot \phi_{i}+\omega_{i}^{*} \cdot \pi_{i}+\gamma_{i}^{*} \cdot \theta_{i}}{\phi_{i}+\pi_{i}+\theta_{i}} & =\frac{\lambda_{i}^{*} \cdot\left(t_{i}^{*} \cdot q_{i}^{*}\right)+\omega_{i}^{*} \cdot\left(\rho_{i}^{*} \cdot q_{i}^{*}\right)+\gamma_{i}^{*} \cdot\left(\sigma_{i}^{*} \cdot q_{i}^{*}\right)}{\left(t_{i}^{*} \cdot q_{i}^{*}\right)+\left(\rho_{i}^{*} \cdot q_{i}^{*}\right)+\left(\sigma_{i}^{*} \cdot q_{i}^{*}\right)} \\
& =\frac{\lambda_{i}^{*} \cdot t_{i}^{*}+\omega_{i}^{*} \cdot \rho_{i}^{*}+\gamma_{i}^{*} \cdot \sigma_{i}^{*}}{t_{i}^{*}+\rho_{i}^{*}+\sigma_{i}^{*}} \\
& \equiv \xi_{i}
\end{aligned}
$$

By substituting (31) into (30), we obtain

$$
\begin{aligned}
L^{*} & =\sum_{i \in \mathrm{I}} \alpha_{i} \cdot \ln \left(q_{i}^{*}+1\right)+\sum_{i \in \mathrm{I}} \xi_{i} \cdot\left\{\left(\phi_{i}+\pi_{i}+\theta_{i}\right)-\left(t_{i}^{*}+\rho_{i}^{*}+\sigma_{i}^{*}\right) \cdot q_{i}^{*}\right\} \\
& =\sum_{i \in \mathrm{I}} \alpha_{i} \cdot \ln \left(q_{i}^{*}+1\right)+\sum_{i \in \mathrm{I}} \xi_{i} \cdot\left(\psi_{i}-p_{i} \cdot q_{i}^{*}\right)
\end{aligned}
$$

By using (11), we obtain $\psi_{i}=\alpha_{i} \cdot \sum_{k \in I} \psi_{k}$ and $p_{i} \cdot q_{i}^{*}=\alpha_{i} \cdot \sum_{k \in I} p_{k} \cdot q_{k}^{*} \forall i \in I$. Then, the second term on the right-hand side of (32) can be transformed as 


$$
\begin{aligned}
\sum_{i \in \mathrm{I}} \xi_{i} \cdot\left(\psi_{i}-p_{i} \cdot q_{i}^{*}\right) & =\sum_{i \in \mathrm{I}} \xi_{i} \cdot\left(\alpha_{i} \cdot \sum_{k \in \mathrm{I}} \psi_{k}-\alpha_{i} \cdot \sum_{k \in \mathrm{I}} p_{k} \cdot q_{k}^{*}\right) \\
& =\sum_{i \in \mathrm{I}} \xi_{i} \cdot \alpha_{i} \cdot\left(\sum_{k \in \mathrm{I}} \psi_{k}-\sum_{k \in \mathrm{I}} p_{k} \cdot q_{k}^{*}\right)
\end{aligned}
$$

Hence, we obtain (34), which is equivalent to (29).

$$
L^{*}=\sum_{i \in \mathrm{I}} \alpha_{i} \cdot \ln \left(q_{i}^{*}+1\right)+\sum_{i \in \mathrm{I}} \xi_{i} \cdot \alpha_{i} \cdot\left(\sum_{k \in \mathrm{I}} \psi_{k}-\sum_{k \in \mathrm{I}} p_{k} \cdot q_{k}^{*}\right)
$$

By regarding $\sum_{i \in \mathrm{I}} \xi_{i} \cdot \alpha_{i}$ in (34) as the optimal Lagrangian multiplier, it is obvious that $L^{*}$ denoted by (34) is obtained by the utility maximization problem, PP-2, at the equilibrium. Hence, it is reasonable to consider that PP-2 is equivalent to PP.

\section{APPENDIX B}

We assume a certain form of travel time function, $t_{a}\left(V_{a}, C_{a}\right)$, in which the explanatory variables are stochastic link flow, $V_{a}$, and stochastic link capacity, $C_{a}$. At first, we consider $t_{a}\left(V_{a}, C_{a}\right) \cdot t_{b}\left(V_{b}, C_{b}\right)$ for the calculation of the covariance of two link travel times. As shown in Uchida (2014, Appendix A), the following relationship can be obtained by performing the $m$ th-order Taylor series expansion to $t_{a}\left(V_{a}, C_{a}\right) \cdot t_{b}\left(V_{b}, C_{b}\right)$ at $V_{a}=v_{a}, V_{b}=v_{b}, C_{a}=c_{a}$ and $C_{b}=c_{b}$.

$$
t_{a}\left(V_{a}, C_{a}\right) \cdot t_{b}\left(V_{b}, C_{b}\right) \approx \sum_{0 \leq i_{1}+j_{1} \leq m} \sum_{0 \leq i_{2}+j_{2} \leq m} b_{i_{1} j_{a}} \cdot b_{i_{2} j_{2} a} \cdot\left(V_{a}-v_{a}\right)^{i_{1}} \cdot\left(V_{b}-v_{b}\right)^{i_{2}} \cdot\left(C_{a}-c_{a}\right)^{j_{1}} \cdot\left(C_{b}-c_{b}\right)^{j_{2}}
$$

where

$$
b_{i_{k} j_{k} a}=\left.\frac{1}{i_{k} ! j_{k} !} \cdot \frac{\partial^{i_{k}+j_{k}} t_{a}\left(V_{a}, C_{a}\right)}{\partial\left(V_{a}\right)^{i_{k}} \cdot\left(C_{a}\right)^{j_{k}}}\right|_{V_{a}=v_{a}, C_{a}=C_{a}} \quad(\text { for } k=1,2)
$$

By assuming that the stochastic link flow and the stochastic link capacities are independently distributed, we obtain

$$
E\left[t_{a}\left(V_{a}, C_{a}\right) \cdot t_{b}\left(V_{b}, C_{b}\right)\right] \approx \sum_{0 \leq i_{1}+j_{1} \leq m} \sum_{0 \leq i_{2}+j_{2} \leq m} b_{i_{1} j_{1} a} \cdot b_{i_{2} j_{2} b} \cdot c_{j_{1} j_{2} a b} \cdot E\left[\left(V_{a}-v_{a}\right)^{i_{1}} \cdot\left(V_{b}-v_{b}\right)^{i_{2}}\right]
$$

where

If $m=1$, (38) is then

$$
c_{j_{1} j_{2} a b}=E\left[\left(C_{a}-c_{a}\right)^{j_{1}} \cdot\left(C_{b}-c_{b}\right)^{j_{2}}\right]
$$

$$
\begin{aligned}
& E\left[t_{a}\left(V_{a}, C_{a}\right) \cdot t_{b}\left(V_{b}, C_{b}\right)\right] \\
\approx & \left\{\left(b_{00 a} \cdot b_{00 b} \cdot c_{00 a b}\right)+\left(b_{00 a} \cdot b_{01 b} \cdot c_{01 a b}\right)+\left(b_{01 a} \cdot b_{00 b} \cdot c_{10 a b}\right)+\left(b_{01 a} \cdot b_{01 b} \cdot c_{11 a b}\right)\right\} \cdot E\left[\left(V_{a}-v_{a}\right)^{0} \cdot\left(V_{b}-v_{b}\right)^{0}\right] \\
+ & \left\{\left(b_{00 a} \cdot b_{10 b} \cdot c_{00 a b}\right)+\left(b_{01 a} \cdot b_{10 b} \cdot c_{10 a b}\right)\right\} \cdot E\left[\left(V_{a}-v_{a}\right)^{0} \cdot\left(V_{b}-v_{b}\right)^{1}\right] \\
+ & \left\{\left(b_{10 a} \cdot b_{00 b} \cdot c_{00 a b}\right)+\left(b_{10 a} \cdot b_{01 b} \cdot c_{01 a b}\right)\right\} \cdot E\left[\left(V_{a}-v_{a}\right)^{0} \cdot\left(V_{b}-v_{b}\right)^{1}\right] \\
+ & b_{10 a} \cdot b_{10 b} \cdot c_{00 a b} \cdot E\left[\left(V_{a}-v_{a}\right)^{1} \cdot\left(V_{b}-v_{b}\right)^{1}\right]
\end{aligned}
$$

Any random variables, $X_{a}$, hold the following two relationships, $E\left[\left(X_{a}-X_{a}\right)\right]=0$ and $\operatorname{cov}\left[X_{a}, X_{b}\right]=E\left[\left(X_{a}-X_{a}\right) \cdot\left(X_{b}-X_{b}\right)\right]$ where $X_{a}=E\left[X_{a}\right]$. Therefore, (39) can be reduced as

$$
\begin{aligned}
& E\left[t_{a}\left(V_{a}, C_{a}\right) \cdot t_{b}\left(V_{b}, C_{b}\right)\right] \\
\approx & \left(b_{00 a} \cdot b_{00 b} \cdot 1\right)+\left(b_{01 a} \cdot b_{01 b} \cdot \operatorname{cov}\left[C_{a}, C_{b}\right]\right)+\left(b_{10 a} \cdot b_{10 b} \cdot 1 \cdot \operatorname{cov}\left[V_{a}, V_{b}\right]\right)
\end{aligned}
$$

In addition, $b_{i_{k} j_{k} a}=t_{a}\left(v_{a}, c_{a}\right)$ if $i_{k}=0$ and $j_{k}=0$, that is, $b_{00 a}=t_{a}\left(v_{a}, c_{a}\right) \forall a \in A$. By substituting (40) and $b_{00 a}=t_{a}\left(v_{a}, c_{a}\right)$ into the definition of covariance of two link travel times shown by (41)

$$
\operatorname{cov}\left[t_{a}\left(V_{a}, C_{a}\right), t_{b}\left(V_{b}, C_{b}\right)\right]=E\left[t_{a}\left(V_{a}, C_{a}\right) \cdot t_{b}\left(V_{b}, C_{b}\right)\right]-E\left[t_{a}\left(V_{a}, C_{a}\right)\right] \cdot E\left[t_{b}\left(V_{b}, C_{b}\right)\right]
$$


we obtain

$$
\begin{aligned}
\operatorname{cov}\left[t_{a}\left(V_{a}, C_{a}\right), t_{b}\left(V_{b}, C_{b}\right)\right] & \approx t_{a}\left(v_{a}, C_{a}\right) \cdot t_{b}\left(v_{b}, c_{b}\right)+\left(b_{01 a} \cdot b_{01 b} \cdot \operatorname{cov}\left[C_{a}, C_{b}\right]\right)+\left(b_{10 a} \cdot b_{10 b} \cdot \operatorname{cov}\left[V_{a}, V_{b}\right]\right) \\
& -E\left[t_{a}\left(V_{a}, C_{a}\right)\right] \cdot E\left[t_{b}\left(V_{b}, C_{b}\right)\right]
\end{aligned}
$$

Next, we consider the term $E\left[t_{a}\left(V_{a}, C_{a}\right)\right]$ in (42). According to Uchida (2014, Appendix A), we have

$$
\left.E\left[t_{a}\left(V_{a}, C_{a}\right)\right] \approx \sum_{0 \leq i+j \leq m} b_{i j a}^{\prime} \cdot c_{j a} \cdot E \mid\left(V_{a}-v_{a}\right)^{i}\right]
$$

where

$$
\begin{gathered}
b_{i j a}^{\prime}=\left.\frac{1}{i ! \cdot j !} \cdot \frac{\partial^{i+j} t_{a}\left(V_{a}, C_{a}\right)}{\partial\left(V_{a}\right)^{i} \cdot\left(C_{a}\right)^{j}}\right|_{V_{a}=v_{a}, C_{a}=c_{a}} \\
c_{j a}=E\left[\left(C_{a}-C_{a}\right)^{j}\right]
\end{gathered}
$$

If $m=1$, we obtain

$$
E\left[t_{a}\left(V_{a}, C_{a}\right)\right] \approx t_{a}\left(v_{a}, c_{a}\right)
$$

Note that, (46) supports the setting of the mean travel time function assumed in the numerical experiment. The same discussion can be applied to stochastic travel cost. By substituting (46) into (42), we obtain

$$
\operatorname{cov}\left[t_{a}\left(V_{a}, C_{a}\right), t_{b}\left(V_{b}, C_{b}\right)\right] \approx\left(b_{01 a} \cdot b_{01 a} \cdot \operatorname{cov}\left[C_{a}, C_{b}\right]\right)+\left(b_{10 a} \cdot b_{10 a} \cdot \operatorname{cov}\left[V_{a}, V_{b}\right]\right)
$$

Following Uchida (2015), the covariance of link flow are given as follows when we regard stochastic demand flow as an independent random variable that follows the same statistical distribution as $\mathrm{O}-\mathrm{D}$ flow.

$$
\operatorname{cov}\left[V_{a}, V_{b}\right]=V M R \cdot v_{a b} \quad \forall a, b \in A
$$

$V M R$ in (48) is the given variance to mean ratio of the random O-D flow. Since we estimate only flows in a traffic assignment problem, $\operatorname{cov}\left[C_{a}, C_{b}\right]$ can be regarded as constant terms. Therefore, it is reasonable to regard the covariance of two link travel times as a function of $v_{a}, v_{b}$ and $v_{a b}$ as shown by

$$
\operatorname{cov}\left[t_{a}\left(V_{a}, C_{a}\right), t_{b}\left(V_{b}, C_{b}\right)\right]=\tilde{\sigma}_{a b}\left(v_{a}, v_{b}, v_{a b}\right)
$$

For simplicity, we assume that the effect of $v_{a}$ on $\operatorname{cov}\left[t_{a}\left(V_{a}, C_{a}\right), t_{b}\left(V_{b}, C_{b}\right)\right]$ is much smaller than that of $v_{a b}$, and therefore (49) could be

$$
\operatorname{cov}\left[t_{a}\left(V_{a}, C_{a}\right), t_{b}\left(V_{b}, C_{b}\right)\right] \approx \tilde{\sigma}_{a b}\left(v_{a b}\right)
$$

Note that, $\operatorname{cov}\left[t_{a}\left(V_{a}, C_{a}\right), t_{b}\left(V_{b}, C_{b}\right)\right]=\operatorname{var}\left[t_{a}\left(V_{a}, C_{a}\right)\right]$ if $a=b$. If we consider a case of $m>1$, then specification of the stochastic distribution of O-D demand and link capacity are required for the calculation of $\operatorname{cov}\left[t_{a}\left(V_{a}, C_{a}\right), t_{b}\left(V_{b}, C_{b}\right)\right]$. In this Appendix, we show a result only when $m=1$. The variance of route travel time is given by (27) because $\operatorname{var}\left[\Xi_{i j}\right\rfloor$ is the sum of the variances of travel time of links that are the members of the set of links for the route plus the sum of the covariance of two travel times for any combination of two different links from the set of links.

\section{REFERENCES}

Becker, G.S., 1965. A theory of the allocation of time. The Economic Journal, Vol. 75, No.299, pp.493-517.

Brownstone, D. \& Small, K. a., 2005. Valuing time and reliability: Assessing the evidence from road pricing demonstrations. Transportation Research Part A: Policy and Practice, Vol. 39, No.4 SPEC. ISSS., pp.279-293.

Chen, A. \& Zhou, Z., 2010. The $\alpha$-reliable mean-excess traffic equilibrium model with stochastic travel times. Transportation Research Part B: Methodological, Vol. 44, No.4, pp.493-513.

Clark, S. \& Watling, D., 2005. Modelling network travel time reliability under stochastic demand. Transportation Research Part B: Methodological, Vol. 39, No.2, pp.119-140.

Cobb, C.W. \& Douglas, P.H., 1928. A Theory of Production. American Economic Review, Vol. 18, No.1, pp.139-165.

Deserpa, A.C., 1973. Comment: Microeconomic Theory and the Valuation of Time: Some Clarification. Regional and Urban Economics, Vol. 2, No.4, pp.401-410. 
Fosgerau, M. \& Engelson, L., 2011. The value of travel time variance. Transportation Research Part B: Methodological, Vol. 45, No.1, pp.1-8.

Fosgerau, M. \& Karlström, A., 2010. The value of reliability. Transportation Research Part B: Methodological, Vol. 44, No.1, pp.38-49.

Fukushima, M., Luo, Z.-Q. \& Pang, J.-S., 1998, A globally convergent sequential quadratic programming algorithm for mathematical programs with linear complementarity constraints, Computational Optimization and Applications, Vol. 10, No.1, pp. 5-34.

Gartner, N.H., 1980. Optimal traffic assignment with elastic demands. Transportation Science, Vol. 14, No.2, pp.174-191.

Hjorth, K. et al., 2015. Estimating exponential scheduling preferences. Transportation Research Part B: Methodological., Vol.81, No.1, pp.230-251.

Jiang, M. \& Morikawa, T., 2004. Theoretical analysis on the variation of value of travel time savings. Transportation Research Part A: Policy and Practice, Vol. 38, No.8, pp.551-571.

Kato, T., Uchida, K. \& Tamura, T., 2013. Estimation of value of travel time and travel time reliability for heterogeneous drivers in road network. The 18th International Conference of Hong Kong Society for Transportation Studies, pp.123-130.

Kidokoro, Y., 2006. Benefit estimation of transport projects-a representative consumer approach. Transportation Research Part B: Methodological, Vol. 40, No.7, pp.521-542.

Kono, T. \& Morisugi, H., 2000. Theoretical examination on value of time for private trips. Journal of Infrastructure Planning and Management, Vol. 639, pp.53-64 (in Japanese).

Lam, T.C. \& Small, K. a., 2001. The value of time and reliability: Measurement from a value pricing experiment. Transportation Research Part E: Logistics and Transportation Review, Vol. 37, No.2-3, pp.231-251.

Lam, W.H.K., Shao, H. \& Sumalee, A., 2008. Modeling impacts of adverse weather conditions on a road network with uncertainties in demand and supply. Transportation Research Part B: Methodological, Vol. 42, No.10, pp.890-910.

Lo, H.K., Luo, X.W. \& Siu, B.W.Y., 2006. Degradable transport network: Travel time budget of travelers with heterogeneous risk aversion. Transportation Research Part B: Methodological, Vol. 40, No.9, pp.792-806.

Lo, H.K. \& Tung, Y.K., 2003. Network with degradable links: Capacity analysis and design. Transportation Research Part B: Methodological, Vol. 37, No.4, pp.345-363.

Ng, M., Szeto, W.Y. \& Travis Waller, S., 2011. Distribution-free travel time reliability assessment with probability inequalities. Transportation Research Part B: Methodological, Vol. 45, No.6, pp.852-866.

Small, K. a., 1982. The scheduling of consumer activities: work trips. American Economic Review, Vol. 72, No.3, pp.467-479.

Sherali, H.D., Narayanan, A., Sivanandan, R., 2003. Estimation of origin-destination trip-tables based on a partial set of traffic link volumes. Transportation Research Part B: Methodological, Vol. 37, No.9, pp.815-836.

Stone, R., 1954. Linear Expenditure Systems and Demand Analysis : An Application to the Pattern of British Demand. The Economic Journal, Vol. 64, No.255, pp.511-527.

Uchida, K., 2012. A study on estimation methods for values of travel time and travel time reliability in road network. Proceedings of the 5th International Symposium on Transportation Network Reliability, pp.65-79.

Uchida, K., 2010. Equilibrium traffic assignment models based on the Co2 emission minimization principle. Selected papers on Environmental Systems Japan Society of Civil Engineers, Vol. 38, p.101-108 (in Japanese).

Uchida, K., 2014. Estimating the value of travel time and of travel time reliability in road networks. Transportation Research Part B: Methodological, Vol. 66, pp.129-147.

Uchida, K., 2015. Travel Time Reliability Estimation Model Using Observed Link Flows in a Road Network. Computer-Aided Civil and Infrastructure Engineering, Vol. 30, No.6, pp.449-463.

Ueda, T., Morisugi, H. \& Hayashiyama, Y., 2002. Some Remarks on Measuring Benefit of Transport Projects. Journal of Institution for Transport Policy Studies, Vol. 5, No.2, p.23-35 (in Japanese).

Varian, H.R., 1992. Microeconomic Analysis (Second Edition). W W Norton \& Co Inc.

Watling, D., 2002. A Second Order Stochastic Network Equilibrium Model, I: Theoretical foundation. 
Transportation Science, Vol. 36, No.2, pp.149-166.

Watling, D., 2006. User equilibrium traffic network assignment with stochastic travel times and late arrival penalty. European Journal of Operational Research, Vol. 175, No.3, pp.1539-1556.

Willig, R., 1976. Consumer's surplus without apology. The American Economic Review, Vol. 66, No.4,pp.589-597. 